\title{
Crítica y participación en la alquimia de la investigación-acción. Una experiencia de formación en convivencia
}

\section{Criticism and participation in the alchemy of action research. An experience of training in coexistence}

\author{
Lucía Elena Rodríguez McKeon ${ }^{1}$ \\ Adalberto Rangel Ruiz de la Peña ${ }^{2}$ \\ Nelly del Pilar Cervera Cobos ${ }^{3}$
}

Recibido: 09/11/20. Aceptado: 15/04/21

http://dx.doi.org/10.30972/dpd.10154817

\section{Resumen}

En este texto se comparten los resultados de una investigación orientada al análisis de los aportes de la investigación-acción en la configuración de diversos dispositivos de innovación en el campo de la gestión de la convivencia, construidos por docentes que laboran en el ámbito de la escuela pública de educación básica en la ciudad de México, dentro de un programa de posgrado, con la finalidad de construir estrategias orientadas a afrontar las problemáticas de convivencia tanto desde el enfoque de los Derechos Humanos y la cultura de paz como desde un enfoque transformativo. A partir del uso de la metodología de análisis documental, se hizo acopio de información relevante respecto al alcance temático, metodológico y didáctico de los dispositivos analizados, a partir de lo cual fue posible identificar la construcción situada, la participación y el ejercicio de la crítica como elementos que fortalecen la configuración de dichos dispositivos desde la investigación-acción.

Palabras clave: investigación-acción; dispositivo de innovación; formación para la convivencia.

\footnotetext{
${ }^{1}$ UPN Correo electrónico: ekeon@upn.mx

${ }^{2}$ UPN Correo electrónico: arangel@upn.mx

${ }^{3}$ UPN Correo electrónico: ncervera@upn.mx
} 


\section{Abstract}

This text shares the results of a research aimed at analyzing the contributions of action research in the configuration of various innovation devices in the field of coexistence management, built by teachers who work in the field of public school of basic education in Mexico City, within a postgraduate program, in order to build strategies aimed at coping with coexistence problems from a human rights perspective and a culture of peace from a transformative approach. From the use of the documentary analysis methodology, relevant information was collected regarding the thematic, methodological and didactic scope of the analyzed devices, from which it was possible to identify the situated construction, the participation and the exercise of criticism. as elements that strengthen the configuration of said devices from action research.

Keywords: Action research, Innovation device, Training for coexistence.

Lo que se comparte en este trabajo forma parte de una investigación más amplia sobre la experiencia de la Maestría en Gestión de la Convivencia en la Escuela. Violencia, Derechos Humanos y Cultura de Paz (MGCE) $)^{4}$ Si bien los resultados obtenidos en dicha indagación refieren a diversas aristas, en este artículo en particular se pone a discusión el aporte del enfoque de la investigación-acción (IA), en su capacidad para dinamizar una alquimia de transformación en las prácticas de la convivencia al favorecer el desarrollo de procesos de crítica, construcción situada y participación que contribuyen a potencializar la configuración de dispositivos de innovación ${ }^{5}$, que muestran su potencialidad para aperturar los principios

\footnotetext{
${ }^{4}$ Este programa se desarrolla por la Universidad Pedagógica Nacional (UPN), en colaboración con la Comisión de Derechos Humanos de la Ciudad de México (CDHCM) desde 2015. Se orienta a la formación de docentes del ámbito de la educación básica y hasta ahora han egresado tres generaciones y una más se encuentra en curso. Sus documentos fundamentales pueden ser consultados en http://mgce.upnvirtual.edu.mx/ Entre otros objetivos, la investigación se ha planteado como propósito identificar y contrastar las principales características de las estrategias de intervención construidas por los estudiantes, buscando comprender a mayor profundidad sus dinámicas de desarrollo, poniendo énfasis en el análisis de las rutas que toman, las tensiones que se presentan y los alcances tenidos. Su desarrollo se ha realizado con el financiamiento otorgado por el Programa de Desarrollo Docente (Prodep) de la Secretaría de Educación Pública de México. Los autores expresan su agradecimiento por dicho apoyo.

${ }^{5}$ El dispositivo de innovación es el trabajo que elaboran los estudiantes que cursan la maestría a lo largo de los cuatro semestres de su duración y que es presentado como tesis para la obtención del grado. Configurado a
} 
que rigen al sistema de relaciones dentro del ámbito educativo, a fin de abonar en la construcción de entornos de convivencia institucionales pacíficos y justos, regidos por el respeto a los Derechos Humanos, asumiendo la importancia que ello reviste para la configuración de espacios escolares posibles de habitar por los niños, niñas y adolescentes que asisten a ellos.

En términos formativos, el programa de la maestría surge con la utopía de configurarse como una especie de laboratorio de innovación que aporte posibilidades al trabajo docente para ensayar propuestas alternativas de tipo amplio y para afrontar las problemáticas de convivencia que hoy se viven en la escuela, desde un enfoque de Derechos Humanos y cultura de paz.

Articulada a la necesidad de enfrentar el desafío de construir otros modos de promover la investigación en su articulación con la intervención, la MGCE surge con la aspiración de convertirse en un lugar de debate y análisis para la construcción de estrategias innovadoras, para imaginar y experimentar diversas alternativas. Dicho proceso toma su concreción en los dispositivos de innovación que son diseñados y desarrollados por los estudiantes a lo largo de los cuatro semestres de duración de la maestría en sus propios espacios de trabajo, a partir del ejercicio de la investigación-acción, que se realiza sustentada en la articulación entre la teoría y la práctica y la reflexión y la acción.

El universo empírico de análisis está constituido por 38 dispositivos de innovación concluidos como tesis de grado hasta ahora, que incluyen algunos elaborados por egresados que cursaron una maestría antecedente en el periodo 2013-2015. Para este análisis se eligieron 25 casos, seleccionados a partir de los criterios de exhaustividad y representatividad propuestos por Goetz y Lecompte (1988), pues interesó que los dispositivos analizados abordaran diversas temáticas y hubieran sido orientados hacia el alumnado directamente, en el contexto de distintos niveles educativos.

partir de preocupaciones en torno a la convivencia en la escuela que son significativas para sus autores, contiene el desarrollo de una aproximación diagnóstica a la problemática de interés, a partir de lo cual se diseñan y desarrollan un conjunto de estrategias de intervención que son acompañadas por el seguimiento de la experiencia. 
A partir del soporte de la metodología de análisis documental, se utilizaron diversos procedimientos para realizar el proceso de reducción de la información de los dispositivos analizados: se consultaron los dispositivos en la página de la maestría, se dio lectura de ellos, se elaboraron resúmenes de las experiencias y se recolectaron datos en función de un esquema específico elaborado previamente. A partir de ello, se analizó e interpretó cualitativamente la información, permitiéndonos obtener una representación de las principales características de los dispositivos analizados, haciendo énfasis en sus alcances conceptuales y metodológicos en relación con los aportes de la investigación-acción en dichos procesos.

\section{La investigación-acción, un eje en la alquimia para la transformación de}

\section{la convivencia}

Ligadas y en vinculación con las comunidades educativas, la perspectiva de la IA pone al centro la participación de los sujetos involucrados en dinámicas de acción-reflexión contextualizadas, a fin de intervenir en la construcción de alternativas frente a los problemas de la práctica.

Siguiendo los planteamientos de Carr y Kemmis (1986), se asume una perspectiva de la investigación-acción que enfatiza su capacidad para promover procesos de reflexión crítica, subrayando su potencialidad para convertirse en el eje de la alquimia ${ }^{6}$ de la transformación, de las prácticas de la gestión de la convivencia en la escuela, al promover formas alternas de producción del conocimiento frente a la racionalidad científica vigente.

A ello se refiere Jara (2009: 3) cuando distingue esta forma de comprender la IA frente a otras opciones:

La IA no ofrece un rosario de pautas, de técnicas, de orientaciones didácticas para aplicar, sino un conjunto de principios ético políticos, epistemológicos y filosóficos que nos llevan a mirar con otros ojos y desde otro lugar, nuestra propia práctica, obligándonos a sumergirnos en las raíces de su sentido y a renovarlas crítica y creadoramente. $Y$ todo ello, como una manera,

\footnotetext{
${ }^{6}$ La noción de alquimia se utiliza de manera metafórica para subrayar el papel que puede jugar la opción metodológica de la investigación-acción cuando ella se articula con dinámicas de transformación de las problemáticas de la convivencia.
} 
tal vez la mejor manera, de formarnos como docentes: hombres y mujeres que nos formamos desde nuestras propias prácticas como sujetos capaces de organizar procesos de construcción de conocimientos, o sea de enseñar, porque desarrollamos nuestras capacidades de aprender.

Asumida en relación con la capacidad racional y emocional de autoemancipación de los seres humanos como actores sociales, la reflexión crítica la definimos con Freire (2006) como la capacidad de leer críticamente el mundo, a fin de develar el ejercicio y los juegos sociales de los poderes que operan de modo estructural en los múltiples ámbitos de vida.

En este contexto, la reflexión crítica implica un hacer político-pedagógico que se nutre por la acción y es opuesto a la adaptación de una realidad sin cuestionarla. Su desarrollo se da cuando se construyen mayores herramientas para interpretar lo concreto, dentro de las espirales acción-reflexión-acción y práctica-teoría-práctica que permiten cuestionar los conocimientos y narrativas e interrogar sobre pensamientos, saberes y acciones que se poseen sobre el campo de la convivencia en la escuela como lugar de formación e intervención.

Ligada al ámbito educativo, la valía del uso de la investigación-acción es que favorece una reflexión individual y colectiva acerca de la relación que se establecen entre los procesos y los productos en circunstancias concretas, lo que constituye, en palabras de Schön (1998), una práctica reflexiva. Lo anterior contribuye, en palabras de Elliot (2005), a la configuración de saberes docentes ligados a sus prácticas al estar guiados por el afán transformativo de ensayar respuestas frente a las problemáticas del hacer.

Es así que su ejercicio propicia que los docentes desarrollen capacidades que les permitan discernir el curso correcto de acción al enfrentarse a situaciones concretas, complejas y problemáticas, favoreciendo el desarrollo de capacidades reflexivas en los docentes, en términos individuales y colectivos, para promover procesos de afrontamiento de los conflictos de modo asertivo con los diferentes actores educativos (estudiantes, pares, padres de familia o autoridades) y la construcción de propuestas de intervención para resolver los problemas de la práctica.

Esta característica que impulsa el trabajo reflexivo y creativo de los participantes, así como su intercambio y cooperación permanente, favorece la toma de conciencia sobre la interdependencia existente entre los elementos que condicionan la génesis y desarrollo de 
las problemáticas y sus procesos de cambio desde la óptica institucional y pedagógica, además de promover el desarrollo de un sentido de agencia colectiva en los docentes que se contraponga a la tendencia de una configuración autorreferenciada y egoísta, como plantean Yurén y Mick (2013), a fin de contribuir en la construcción de un sentido de valores y opciones consistentes y cohesivas como comunidades educativas al asumir su responsabilidad en la transformación de las realidades escolares. En este sentido, la investigación-acción enlaza el proceso de la investigación educativa, en tanto proceso cíclico y reflexivo, con la práctica educativa ligado a la transformación educativa.

\section{La construcción situada, la participación y la crítica: tres elementos que se}

\section{producen en la alquimia de la investigación-acción}

Al realizar el análisis de lo que aporta la investigación-acción en la construcción de los dispositivos de innovación para abrir la forma de lo escolar en torno a la convivencia, identificamos que esta opción metodológica favorece el desarrollo de diversos procesos, entre los que destacan: el ejercicio de la crítica, la construcción situada y la promoción de procesos reales de participación, como se compartirá a continuación.

\subsection{La construcción situada y la participación}

A partir del análisis encontramos que la investigación-acción constituye una herramienta fundamental que fortalece el engranaje metodológico de la MGCE para favorecer la construcción situada de alternativas frente a las problemáticas de la convivencia. El esfuerzo está en pensar desde lo específico para nutrir el desarrollo de propuestas contextualmente significativas al conectarse con lo particular.

Frente a la tendencia a construir propuestas que dan respuestas únicas, homogéneas y generalizantes frente a problemáticas diversas y complejas, que poco involucran a los sujetos y tampoco se vinculan con las problemáticas de la práctica, por lo que resultan insuficientes para abordar y dar respuesta a las problemáticas cotidianas que se viven en las escuelas, el enfoque de la IA contribuye a construir otras rutas al plantearse la intervención desde el contexto en situación, debido a que lo que interesa es que sean los sujetos quienes piensen 
sus problemas, a fin de crear alternativas innovadoras que pongan al centro la intención de promover procesos situados de cambio estructural, más que de continuidad educativa. (Boggino y Rosekrans, 2004).

En este sentido, construir desde lo situado, contribuye a trascender la generalización autoritaria que mira todo igual para hacer la pregunta desde lo singular. Construir desde lo situado es hacerlo con y desde la perspectiva de los sujetos. Así se observa en las producciones diagnósticas que adquieren un lugar central dentro de los dispositivos analizados.

Frente al afán generalizado de enjuiciar la realidad desde una normatividad modélica o que se basa en el análisis desde la superficialidad de situaciones que requieren procesos reflexivos más amplios, en la mayoría de los diagnósticos que sustentan la construcción de los dispositivos se hace uso de diversos instrumentos y recursos de tipo cualitativo que profundizan en la comprensión de las problemáticas de intervención al promover su inmersión en ellas.

Entrevistas, grupos focales y observación resultan ser algunos de los instrumentos más utilizados para acercarse a las perspectivas de los sujetos, acompañados de otros recursos que buscan construir situaciones de comunicación y escucha entre los sujetos involucrados en el desarrollo de los proyectos como punto de partida para la identificación y análisis de las problemáticas, al asumir que uno de los grandes desafíos del desarrollo de los proyectos en el terreno de la práctica se encuentra relacionada con la posibilidad de generar dinámicas donde la comunidad escolar asuma un lugar protagónico para pensar los problemas e idear alternativas novedosas frente a ellos.

Ahora bien, poner el foco en la comprensión del contexto situado como eje en la construcción de los dispositivos de innovación no significa solamente hacer referencia a los referentes compartidos. Un esfuerzo en los diagnósticos está dado por escuchar y documentar la diversidad de voces que desde posiciones distintas poseen perspectivas conflictivas entre sí, como pudo documentarse en los dispositivos donde los problemas son analizados desde el punto de vista del estudiantado o de los padres y madres de familia, en contraposición con la perspectiva de los propios docentes. 
En este sentido, desde la IA es posible incentivar la escucha sobre las distintas perspectivas implicadas de quienes participan en los problemas de convivencia en la escuela, asumiendo que estos pueden ser vividos de manera diferente, según la posición que ocupan los diversos actores involucrados al interior de la estructura escolar. Este aspecto resulta relevante debido a que, al poner el foco de análisis en la comprensión de los puntos de vista encontrados, se pone atención a las tensiones que ello genera, asumiendo que la convivencia se encuentra signada vitalmente por la discrepancia y el conflicto.

Ligado a lo anterior, la promoción de procesos reales de participación en el sentido planteado por Hart (1993) constituye un elemento concomitante a los procesos de construcción situada de los dispositivos de innovación.

Al participar en la construcción de los dispositivos de innovación, los sujetos -tanto maestros como alumnos- experimentan la posibilidad de configurar un sentido de agencia colectiva que les permite reconocerse como actores con eficacia política que pueden hacerse cargo, al desarrollar proyectos compartidos a partir del convencimiento de que ellos y ellas pueden y son responsables de intervenir en la construcción del orden social de la escuela.

Ello contribuye a la fractura del principio de jerarquía basado en el adultocentrismo como elemento básico que rige al sistema de relaciones entre maestros y alumnos que asegura la no-participación dentro del ámbito educativo, abriendo el espacio para la construcción de relaciones más horizontales entre ellos, más allá de los lugares asignados tradicionalmente.

Este desplazamiento que reconoce a docentes y estudiantes desde una nueva perspectiva tiene efectos en la promoción de estrategias de trabajo colaborativo entre ambos que inauguran la inclusión de nuevas prácticas para resolver los problemas que desafían a la convivencia en el aula y la escuela, tales como la construcción de acuerdos y reglas compartidas, el debate y la deliberación, y las estrategias para el desarrollo de capacidades de afrontamiento de los conflictos que favorecen otras formas de convivencia en el aula y la escuela, al quedar entramadas en las dinámicas de trabajo educativo cotidiano como estrategias estructurantes.

Todo ello abre condiciones para el desarrollo de procesos de expansión formativa (Cox, 2010) que amplían los márgenes de intervención de los sujetos en los procesos de la 
formación para la convivencia, al romper con la limitación implicada en la consideración de que los contenidos educativos en la formación para la convivencia se encuentran encerrados en el código curricular que se transmite en el aula, dejando ver la existencia de la dimensión ético política ${ }^{7}$ por donde transcurre la formación que se abre hacia el espacio de la escuela y la familia como lugares fundamentales para aprender formas alternas de convivir, como se pudo observar en algunos dispositivos orientados a construir estructuras de comunicación, participación y reconocimiento en el aula y la escuela que contribuyeron a desnaturalizar la violencia y construir formas de regulación de la convivencia basados en la cultura de paz.

Este último aspecto es significativo, pues amplía el abanico de posibilidades para la intervención didáctica y pedagógica más allá del curriculum formal, lo que fractura la forma escolar dominante, basada en la separación entre lo que se vive y se aprende, poniendo en cuestión la centralidad del currículo como organizador de un proceso educativo limitado a la prescripción de un conjunto de contenidos educativos que mediatizan, ocultan y se oponen al carácter educativo de la vida misma, al sobreponerse a la experiencia educativa en el campo de la convivencia que se sucede en el plano de las relaciones sociales en el ámbito de la escuela (Rodríguez, 2009).

\subsection{La crítica}

La espiral de transformación que se desata a partir de la consideración de que solo cuando comprendemos cómo es que se configuró algo es posible desmontar el conjunto de elementos que pueden condicionar que otra situación sea posible, es otro de los aportes de la IA en la construcción de los dispositivos.

\footnotetext{
${ }^{7}$ La dimensión ético-política de la formación involucra el desarrollo de múltiples aprendizajes que influyen en la manera de ser, de actuar y de relacionarse que se desarrollan de manera en muchos casos, implícita, aunque no sin intencionalidad como experiencias ligadas a los procesos de socialización política. Su espacio de desarrollo deriva más del contenido pedagógico que se produce en las relaciones que se establezcan con y en la institución educativa (Rodríguez, 2009). En el campo de la formación para la convivencia su eficacia es alta, en tanto que conlleva una movilización disposicional completa del sujeto, que involucra no solo a la dimensión epistémica y técnica, sino también la ética y existencial, siguiendo el planteamiento de Yuren (2005).
} 
A partir del análisis, identificamos que la crítica se convierte en una clave fundamental que propicia el ejercicio de pensar lo impensado (Freire, 2004), como momento fundante en la construcción de otras vías posibles para la convivencia en la construcción de los dispositivos.

Este aspecto es relevante debido a que resulta necesario tener presente que los dispositivos de intervención en el campo de la convivencia son espacios de producción de sentidos y prácticas articuladas a preocupaciones pedagógicas que condicionarán sus límites y posibilidades para afectar el orden de relaciones en la escuela, por lo que resulta necesario promover el desarrollo de la capacidad de reflexión crítica para comprender las problemáticas de la convivencia desde perspectivas más profundas y complejas, como condición para la construcción de respuestas situadas.

Por un lado, llama la atención el proceso de deconstrucción de las interpretaciones que los docentes llevan a cabo respecto a lo que les preocupa de la convivencia en su práctica educativa, al producirse un desplazamiento en la forma de comprender los factores que explican los problemas de la convivencia, como se puede ver en la siguiente tabla.

Tabla 1. La reconfiguración de la perspectiva de análisis sobre los problemas de convivencia

\begin{tabular}{|c|c|}
\hline \multicolumn{2}{|c|}{} \\
\hline $\begin{array}{c}\text { Del foco en la mirada criminalizante } \\
\text { del alumno problemático considerado } \\
\text { como culpable. }\end{array}$ & $\begin{array}{c}\text { Hacia el análisis de la forma como las } \\
\text { estructuras escolares condicionan el } \\
\text { desarrollo de dichas situaciones. }\end{array}$ \\
\hline $\begin{array}{l}\text { Del foco en la mirada que patologiza } \\
\text { al estudiante con discapacidad como } \\
\text { sujeto incapaz. }\end{array}$ & $\begin{array}{l}\text { Al reconocimiento de su potencial de } \\
\text { aprendizaje que es condicionado por las } \\
\text { dinámicas grupales que actúan como } \\
\text { posibles barreras. }\end{array}$ \\
\hline $\begin{array}{l}\text { De la mirada que considera que el } \\
\text { afuera de la escuela produce de manera } \\
\text { unilateral las problemáticas de de } \\
\text { convivencia a su interior. }\end{array}$ & $\begin{array}{l}\text { A la configuración de una mirada amplia y } \\
\text { compleja sobre la interrelación del adentro y } \\
\text { eluera en la configuración de las } \\
\text { problemáticas de la convivencia escolar. }\end{array}$ \\
\hline
\end{tabular}


De la mirada que explica que los problemas de disrupción y/o dificultades de regulación son culpa de los alumnos que no respetan la disciplina.
Al reconocimiento de la forma como intervienen los distintos actores en la configuración de un clima que permita lidiar con las situaciones problemáticas que se presentan en la convivencia.

La mirada inquisidora que se propicia en el momento del diagnóstico situado desde la investigación-acción favorece procesos dialógicos-reflexivos que de manera crítica interpelan y ponen en cuestión las visiones dominantes en la forma de representar los problemas de la convivencia, respaldando la ruptura con aquellas explicaciones limitadas que sobredimensionan la presencia de los comportamientos de ciertos alumnos que son estigmatizados como los culpables de dichas problemáticas.

Articulados a la racionalidad pedagógica vigente, es sabido que la forma como se explican los problemas de la convivencia en las escuelas han sobredimensionado una perspectiva que tiende a valorar que dichas manifestaciones son productos externos a la escuela y donde prevalecen miradas estigmatizantes que nublan la posibilidad de comprender a cabalidad los factores implicados en dichas problemáticas, produciendo una mirada simplista y fragmentada que impide construir alternativas con una mayor potencialidad para propiciar cambios de manera más integral, por lo que resulta relevante el proceso de deslizamiento que se identifica en la forma de comprender las problemáticas desde otras perspectivas que reconocen las múltiples relaciones implicadas de manera dialéctica y compleja en ellas. Esta dinámica se convierte en el espacio que anuda un proceso de análisis y toma de conciencia como condición y proceso para el cambio.

Relacionado con lo anterior, otro aspecto identificado en el análisis se refiere a la amplitud de temáticas que emergen en la construcción de los dispositivos en el proceso de formación de la maestría, lo que deja ver la toma de conciencia sobre la diversidad de planos 
que de manera articulada intervienen de manera compleja en la génesis y desarrollo de los problemas de convivencia que se configuran en la escuela, tal como se puede ver en la siguiente figura.

Figura 1. Abanico de rutas para afrontar las problemáticas de la gestión de la convivencia

Inclusión

No discriminación

Reconocimiento de la diferencia

\section{Visión holística} articulaciones y tejidos entre saberes y prácticas

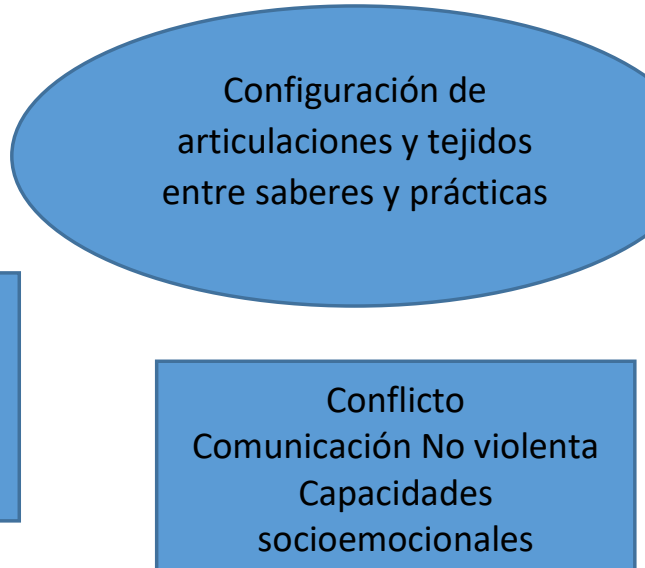

Colaboración Participación Normas y justicia restaurativa

Sin el afán de realizar una clasificación rígida de los campos temáticos que se identifican en los dispositivos elaborados por los egresados de la maestría, debido a los riesgos que dicha práctica entraña en términos del oscurecimiento de la posibilidad de comprender la singularidad de cada una de las experiencias, encontramos que las propuestas construidas focalizan sus esfuerzos en las nociones de conflicto, inclusión y participación como tres grandes nociones clave que articulan los ejes temáticos, alrededor de los cuales se ensayan vías para construir alternativas de educación para la paz transformativa: desde el desarrollo de capacidades para afrontar conflictos de manera pacífica, la mejora de los procesos de comunicación y la formación de capacidades socioemocionales como contenidos tradicionales de la educación para la paz hasta el desarrollo de experiencias orientadas a combatir la discriminación y promover el reconocimiento de la diferencia y procesos reales 
de inclusión, además de propuestas que incursionan en la construcción de un clima de trabajo que concitan la participación, el trabajo colaborativo y el desarrollo de la autonomía en los sujetos como rasgos que impregnan el trabajo y las relaciones educativas como condición para el desarrollo de disposiciones y capacidades para vivir la cultura de paz. Enfocados, ya sea al trabajo con los estudiantes o dirigidos a los docentes con la intención de construir otras formas de relación, la relevancia de la amplitud temática sobre la cual se construyen los dispositivos de innovación es que dejan ver la configuración de una mirada holística sobre el objeto de la convivencia en la escuela a partir de lo cual es factible la construcción de alternativas de tipo más integral basadas en las articulaciones, como se comparte a continuación.

\section{a) Dispositivos que abordan el conflicto, lo socioemocional y la comunicación no violenta}

En este campo se agrupan 11 dispositivos que promueven el desarrollo de capacidades específicas para lidiar de manera pacífica con el afrontamiento del conflicto. Cuatro estrategias se orientan al desarrollo de capacidades en las y los estudiantes para la negociación y mediación de los conflictos a través del uso de diversas herramientas y recursos relacionados con el diálogo y la búsqueda de acuerdos. Otros cuatro dispositivos se enfocan en el desarrollo de capacidades socioemocionales y dos más subrayan los procesos de comunicación no violenta en la convivencia. A continuación, se comparten los rasgos de algunos de ellos.

El dispositivo "El rincón del diálogo" (Sánchez, 2015) fue organizado como espacio donde los estudiantes de preescolar desarrollan prácticas de diálogo, negociación y búsqueda de acuerdos para construir alternativas compartidas frente a los conflictos cotidianos que surgen en la convivencia diaria, apoyados del trabajo de asambleas para exponer los problemas y del aprendizaje de herramientas para el análisis y mediación de los conflictos.

Otra experiencia destacable en este ámbito es el dispositivo "Protocolo de atención a padres y madres de familia" (Luna, 2019), que ha tenido como propósito promover el manejo asertivo de las situaciones de conflicto escolar que se presentan entre la gestión directiva de 
la escuela y los padres de familia. A partir del uso de herramientas comunicativas desde el enfoque de la cultura de paz, se construyen estrategias para lidiar con las tensiones que genera la convivencia y construir nuevas relaciones libres de violencia, frente a la tendencia al enfrentamiento cotidiano que ha caracterizado la compleja relación entre ambos actores dentro de las escuelas públicas mexicanas.

Por otro lado, la propuesta "Puentes de mediación" (Ugalde, 2017) se orienta a promover procesos de sensibilización y formación en el estudiantado que contribuyan al desarrollo de sus propias herramientas para promover la resolución de sus conflictos de manera más horizontal a través de procesos de mediación entre pares. Entre los aportes significativos de la experiencia, destaca el desarrollo de actividades para construir espacios de confianza entre el alumnado a través de herramientas socioafectivas, así como su énfasis en propiciar que los estudiantes se asuman como sujetos protagónicos que desarrollan sus capacidades para resolver por sí mismos sus conflictos en la esfera cotidiana, dejando de depender de la intervención de los docentes para afrontarlos.

Las cuatro experiencias que profundizan en el desarrollo de capacidades de tipo socioemocional (Cuevas, 2015; García, 2017; Solís, 2017; Ramírez, 2020) se han construido para alumnado de preescolar y primaria, y tres de ellas fueron coordinadas por las docentes de grupo en la generación 2017-2019, coincidiendo con el auge que tomó la temática dentro de los planes de estudio de la educación básica publicados en 2017. Su valor estriba en que permite construir alternativas que articulan de manera significativa los contenidos de educación socioemocional de los planes y programas de estudio con el desarrollo de capacidades para afrontar los conflictos que se viven dentro de sus contextos de vida, a fin de ir más allá del carácter retórico que adquieren este tipo de temáticas cuando se trabajan en sí mismas.

Por último, los dos trabajos titulados "La comunicación y el reconocimiento del otro en los procesos de convivencia" (Rodríguez, 2016) y "Deconstrucción de la risa en el contexto escolar" (Rojas, 2017) aportan elementos muy sugerentes sobre la relación entre las formas de comunicación del estudiantado y la educación para la paz. El primer trabajo pone al centro la preocupación sobre la desnaturalización de la violencia como fenómeno social 
naturalizado en la relación entre las y los estudiantes, poniendo especial atención a la forma como ello se manifiesta en las formas de comunicación violenta en la convivencia. Partiendo de una perspectiva que hace mención de la importancia de la comunicación con un sentido proposicional verdadero, se problematiza la relación existente entre la verticalidad en las relaciones y las formas de comunicación violenta, al asumir que estas últimas se encuentran atravesadas por dinámicas de poder. A partir de ello, se construye una estrategia que pone al servicio de los estudiantes el uso del micrófono escolar, a fin de compartir mensajes que son el medio para hablar sobre los temas que son de importancia para la comunidad estudiantil en el recreo escolar como espacio público de diálogo y deliberación.

El segundo trabajo trata un aspecto muy invisibilizado en la relación entre el estudiantado en secundaria, referido al papel humillante y cruel de la risa como detonador de la violencia, a partir de lo cual se construye un conjunto de estrategias para aperturar una mirada deconstructiva sensible y comprometida hacia la risa, con un enfoque ético que permita disminuir los picos de violencia directa entre las y los jóvenes, desde una mirada transformacional del conflicto, inserta en el paradigma de la construcción de una cultura de paz positiva.

\section{b) Inclusión, no discriminación y reconocimiento de la diferencia}

En relación con los temas de discriminación y reconocimiento de la diferencia que se articulan a partir de la noción de inclusión, se han producido también 11 dispositivos enfocados a problematizar la naturalización de las dinámicas de estigmatización hacia algunos estudiantes que justifican los estereotipos y prejuicios que se promueven dentro del aula como formas naturalizadas de violencia, a partir de lo cual los diversos dispositivos incursionarán por diversas rutas en la construcción de estrategias que promuevan condiciones para la inclusión de todas y todos los estudiantes, independientemente de su condición económica, cultural, de género, étnica, de capacidades y/o etaria.

La valía de incursionar en las problemáticas de la discriminación, desde la educación para la paz, es que hace posible intervenir las situaciones de rechazo entre el estudiantado desde una perspectiva socioestructural que visibiliza la relación existente entre violencia cultural y 
exclusión, superando la visión que sobredimensiona los factores de tipo individual y/o interpersonales en su configuración.

Esta visión sobre el fenómeno permitirá poner énfasis en la identificación de las formas como se construyen los márgenes de inclusión-exclusión en la escuela, asumiendo que ellos condicionan formas de reconocimiento que harán plausible la construcción de un clima de convivencia pacífico y democrático, siempre y cuando se visibilice el derecho a la no discriminación como principio de regulación de la convivencia, desde el marco de los Derechos Humanos.

Este último aspecto se identifica en la orientación que toman los trabajos "El uso del Facebook para atender el tema de la discriminación con alumnos de segundo grado de secundaria" (Romero, 2017), "Intervención educativa para favorecer una convivencia pacífica e inclusiva: procesos y tendencias de cambio" (Rodríguez, 2019) y "Transformando la discriminación y exclusión entre alumnas y alumnos de primer grado de secundaria" (Cedillo, 2019). En ellos se plantea con claridad la necesidad de comprender la dinámica que adquiere el fenómeno de la discriminación y las bases de tipo estructural y cultural en las que se sustenta como forma de violencia, siguiendo el planteamiento de Galtung (1998), como el punto de partida para el diseño de un conjunto de estrategias de intervención.

El trabajo "Los procesos de inclusión-exclusión en las interacciones entre estudiantes dentro de la convivencia escolar", cuya autora es Verónica Rivas (2015), sigue una ruta similar, aunque hace énfasis en la necesidad de promover el reconocimiento del otro en su diferencia como sujeto con dignidad y derechos, sustentada en los aportes de la pedagogía de la alteridad, siguiendo los planteamientos filosóficos de Lévinas (1961) y Skliar (2017).

Otro ámbito de problematización de las dinámicas de discriminación y exclusión desde el enfoque de la cultura de paz es el referido a la problemática que vive el alumnado con discapacidad en los procesos de integración educativa. En los dispositivos "Aprendiendo a convivir, una experiencia de inclusión en el CAM" (Sotelo, 2019) y "La enseñanza de las habilidades socioemocionales en el contexto de la educación inclusiva en la escuela regular" (González, 2018) se analizan las dificultades de hacer realidad el derecho a la inclusión de la población estudiantil con discapacidad. 
A partir de la crítica que se realiza a la mirada patologizante que impide los procesos de inclusión real de los sujetos con discapacidad, ambos dispositivos plantean la necesidad de construir otra mirada sobre el problema que deje de estar enfocada solamente en las problemáticas del niño con barreras, para centrarse en la construcción de las condiciones necesarias para su participación al interior de las dinámicas del grupo escolar.

En este orden de ideas, se subraya la crítica hacia aquellas explicaciones que sobredimensionan la determinación de los factores de orden individual que justifican las concepciones que amparan su tutela como sujetos incapaces, para dar paso a su reconocimiento como sujetos con derechos y potencialidades, para aprender a su ritmo y así participar en la construcción del orden social de la escuela.

Por otro lado, cabe mencionar que tres dispositivos han focalizado en los procesos de discriminación por razones de género. A través de una diversidad de estrategias y actividades, han favorecido el cuestionamiento de las identidades binarias de género, el ejercicio acrítico de las masculinidades hegemónicas, a la vez que han promovido el reconocimiento de las diversidades sexuales.

Finalmente, es de destacar otra vía incursionada por dos dispositivos, referida a la problematización del sentido heterónomo que limita los procesos de formación escolar. Esta perspectiva, en conjunto con los dispositivos construidos por los egresados que se orientan al trabajo con docentes a fin de promover el cuestionamiento de las bases adultocéntricas que explican la crisis por la que atraviesa la relación educativa entre maestros y alumnos en las escuelas, abre otro campo de innovación que propone el desarrollo de múltiples actividades para propiciar el desarrollo de autonomía en el estudiantado, así como el trabajo de sensibilización y formación con el colectivo docente, como estrategias para poner las bases de una educación para la paz transformativa.

\section{c) La construcción de un clima pacífico. Una mirada holística de los procesos de intervención}


Otra perspectiva que se pudo identificar en algunos de los dispositivos analizados se refiere a la construcción de procesos de intervención que enfatizan la necesidad de construir un clima pacífico de convivencia como vía para el desarrollo de la cultura de paz.

La característica que une a dichas propuestas se caracteriza por la construcción de estrategias que favorecen la articulación de diversos recursos que se vinculan al trabajo cotidiano que se realiza en el aula y la escuela. Varias actividades y estrategias se plantean como un conjunto de procesos orientados a la construcción de climas sociales escolares favorables.

Actividades orientadas a -en las que se desarrollan prácticas pedagógicas que facilitan la integración y participación de los alumnos- permiten promover la comunicación, el diálogo, la participación individual y colectiva, con un enfoque de Derechos Humanos y cultura de paz, articulados directamente con las prácticas de enseñanza y de aprendizaje, todo ello con la finalidad de afrontar el desafío de favorecer procesos de integración grupal que vayan en contra del rechazo y desconocimiento del otro, y disminuyan la frecuencia de situaciones violentas, tanto en lo que respecta al hostigamiento como a las manifestaciones de violencia directa, propiamente dicha.

Estos dispositivos han sido desarrollados fundamentalmente por docentes frente a grupo y por una directora de escuela que tienen la posibilidad de realizar procesos de intervención más continuados a los que pueden dar seguimiento en el tiempo.

Desarrolladas por docentes de primaria frente a grupo y una directora de escuela, la noción de espacio juega un papel central en la conceptualización en estas propuestas, como lugar donde se generan los encuentros entre los sujetos mediados por estructuras de tipo material y simbólico que condicionan las posibilidades y límites de la comunicación, la integración y la participación de los sujetos, como características básicas en la construcción de un sentido de comunidad, que permita favorecer procesos de cohesión y reconstrucción del tejido social en el espacio escolar como eje central en la construcción de la cultura de paz.

Planteados como el desarrollo de un conjunto de estrategias que irrumpen en la cotidianidad de la comunidad y sujetos participantes, la valía de estos dispositivos es que se 
proponen modificar el espacio social como el objetivo central de la intervención, partiendo de que a su interior sucede la vida de la comunidad articulada a ciertos principios que reproducen la cultura de la violencia, por lo que los cambios de mayor o menor escala siempre podrán afectar el desarrollo de las prácticas habituales de los sujetos en un contexto determinado.

Es el caso de las propuestas "La integración grupal en la configuración de la convivencia: un caso desde la escuela primaria" (Pérez, 2017), "El trabajo colaborativo entre profesores y alumnos como medio para generar una mejor convivencia" (Ortiz, 2017) y "Estrategias docentes ante los comportamientos disruptivos en el aula" (Morales, 2017). En dichos dispositivos, los docentes ponen al centro el desarrollo de estrategias colaborativas y de trabajo en equipo, hacen uso de la asamblea y la construcción colectiva de cómo mecanismos que regulan la convivencia permiten dar cauce a los desacuerdos y conflictos que de manera cotidiana se viven en el contexto del aula, las que se desarrollan apoyadas en el uso del juego, del arte, además del énfasis en la realización de cambios en la propia disposición material del espacio.

Por otro lado, es de destacar la propuesta "Educación para la paz, el camino para la transformación" (Rodríguez, 2015). El trabajo que se realiza hace uso de los espacios compartidos en la escuela, favoreciendo que los estudiantes de los diversos grados escolares establezcan contacto, se conozcan entre sí, además de favorecer el trabajo de colaboración y tutoreo para la realización de proyectos comunes basados en el juego que se realizan en el momento del recreo activo, lo que contribuye a fortalecer la cohesión de las y los estudiantes en el ámbito de la escuela. Esta propuesta coincide con los dispositivos diseñados por las dos directoras de escuela primaria que por su posición logran incentivar la participación de los distintos actores docentes de la escuela para actuar de manera conjunta en el desarrollo de un proyecto que involucra a la totalidad de la comunidad escolar, potenciando los resultados de dichas intervenciones en la construcción de ambientes pacíficos como espacios para el desarrollo de procesos de cultura de paz.

\section{Algunas reflexiones finales}


A partir del análisis realizado, es posible apuntar algunas reflexiones finales como punto de partida para continuar con el análisis de la contribución de la investigación-acción a la experiencia de formación de la MGCE y sus resultados.

Por un lado, la IA es el modelo de investigación pertinente para impulsar los procesos de formación de docentes en programas de esta naturaleza, debido a que contribuye a su articulación con las problemáticas de la práctica, permitiendo la construcción de alternativas situadas que responden a los desafíos que presentan los contextos concretos de las escuelas.

Por otro lado, inserta en una perspectiva que destaca su carácter dialógico reflexivo, esta opción metodológica favorece el desarrollo de una perspectiva crítica para el impulso de procesos de cambio, que acompañados por dinámicas participativas reales contribuyen a la apertura de lo posible de pensar y hacer en el ámbito de la escuela.

Por otro lado, esta orientación que favorece la intervención en prácticas que se desenvuelven en los contextos de las realidades locales propician procesos de aprendizaje abierto que privilegian las articulaciones y los tejidos entre el saber y el actuar, y entre la reflexión y la práctica, como vía para la generación y aplicación del conocimiento socialmente útil, a partir del cuestionamiento de los paradigmas tradicionales de la gestión de la convivencia, desde una perspectiva crítica.

Para finalizar, es necesario subrayar que vista desde la dimensión ético-política, la inclusión de la investigación-acción insta a los actores educativos a ver más allá de sí mismos, dejando de privilegiar el interés propio, propiciando su configuración como individuos activos en la conformación del poder, el gobierno, la igualdad y la justicia. El proceso de formación desarrolla así la configuración de un sentido de agencia moral y política, utilizando la capacidad de análisis crítico y cultivando una sensibilidad ética a través del cual aprender a respetar los derechos de los demás, como plantea Giroux (2019).

En este sentido, la investigación-acción abre condiciones para fracturar la forma de lo escolar, promoviendo la reconstrucción de comunidades educativas bajo otros principios, que desplazan el trabajo ligado al desarrollo de una individualidad hacia la búsqueda de la colaboración y solidaridad. 


\section{Bibliografía}

Bárcenas, R. (2019) El afrontamiento de conflictos en la escuela secundaria. Una experiencia de aprendizaje en estudiantes de secundaria. Tesis de maestría. México: Universidad Pedagógica Nacional.

Bernard, M. (2004). Arquitectura y dispositivos de formación. En Castañeda, A.; Navia, C. y Yurén, T. (coords.) Formación, distancias y subjetividades. Nuevos retos de la formación en la globalización. México: Limusa.

Boggino, N. y Rosekrans, K. (2004). Investigación acción. Reflexión crítica sobre la práctica educativa. Buenos Aires: Homo Sapiens.

Carr, W. y Kemmis, S. (1986). Teoría crítica de la enseñanza. La Investigación acción en la formación del profesorado. Barcelona: Martínez Roca.

Cedillo, K. (2019). Transformando la discriminación y exclusión entre alumnas y alumnos de primer grado de secundaria: su diagnóstico, intervención y alcances educativos. Tesis de maestría. México: Universidad Pedagógica Nacional.

Cox, C. (2014). Educación ciudadana en América Latina: Prioridades de los currículos escolares. Ginebra: IBE Unesco.

Cuevas, I. (2017). El desarrollo de competencias emocionales para la gestión de la Convivencia. Tesis de maestría. México: Universidad Pedagógica Nacional.

Elliot, J. (1993). El cambio educativo desde la Investigación Acción. Madrid: Morata.

Freire, P. (2004). Pedagogía del Oprimido. México: Siglo XXI.

Estébes, M. (2016). Estrategia de intervención para problematizar los estereotipos de género y promover el desarrollo de capacidades para una convivencia equitativa entre hombres y mujeres. Tesis de maestría. México: Universidad Pedagógica Nacional.

Galtung, J. (1998). Tras la violencia, 3R: reconstrucción, reconciliación, resolución. Bilbao: Ed. Gorgoratuz.

Giroux, H. (2019). Hacia una pedagogía de la esperanza educada bajo el capitalismo de casino (Trad A. Micán). Pedagogía y Saberes, 50, 153-158. 
García, M. (2019). La promoción de habilidades socioemocionales desde el juego cooperativo y la educación física. Tesis de maestría. México: Universidad Pedagógica Nacional.

Goetz, J.P. y Lecompte, M. (1988). Etnografía y diseño cualitativo en investigación educativa. Madrid: Morata.

Gonzáles, M. (2018). La enseñanza de las habilidades socioemocionales en el contexto de la educación inclusiva en la escuela regular. Tesis de maestría. México: Universidad Pedagógica Nacional.

Hart, R. (1993). La participación de los niños: de la participación simbólica a la participación auténtica. Colombia: Fondo de Naciones Unidas para la Infancia (Unicef-Orealc). Ensayos Innocenti, $4 . \quad$ Disponible en: http://www.unicefirc.org/publications/pdf/ie participation spa.pdf

Jara, O. (2009). El desafío y la pasión por aprender. Revista Rizoma Freiriano, 4.

Luna, G. (2019). Atención y resolución de conflictos escolares desde el enfoque de la cultura de paz con padres de familia. Tesis de maestría. México: Universidad Pedagógica Nacional.

Monter, N. (2019). Dinámicas de convivencia entre estudiantes de secundaria. Repensando los roles y estereotipos de género. Tesis de maestría. México: Universidad Pedagógica Nacional.

Morales, M. (2017). Estrategias docentes ante los comportamientos disruptivos en el aula. Tesis de maestría. México: Universidad Pedagógica Nacional.

Ortiz, A. (2017) El trabajo colaborativo entre profesores y alumnos como medio para generar una mejor convivencia. Tesis de maestría. México: Universidad Pedagógica Nacional.

Pérez, O. (2017). La integración grupal en la configuración de la convivencia: un caso desde la escuela primaria. Tesis de maestría. México: Universidad Pedagógica Nacional.

Polo, G. (2017). Jóvenes y la autonomía por venir. Cultura de paz y derechos humanos. Tesis de maestría. México: Universidad Pedagógica Nacional.

Ramírez, B. (2020). Aprendiendo a convivir: la importancia de la regulación emocional en la educación preescolar. Tesis de maestría. México: Universidad Pedagógica Nacional. 
Ramírez, L. (2019). El aprendizaje social como forma de disminuir la violencia en la escuela. Tesis de maestría. México: Universidad Pedagógica Nacional.

Rivas, V. (2015). Los procesos de inclusión-exclusión en las interacciones entre estudiantes dentro de la convivencia escolar. Tesis de maestría. México: Universidad Pedagógica Nacional.

Rodríguez, A. (2016). La comunicación y el reconocimiento del otro en los procesos de convivencia. Tesis de maestría. México: Universidad Pedagógica Nacional.

Rodríguez, R. (2016). Educación para la paz, el camino para la transformación. Tesis de maestría. México: Universidad Pedagógica Nacional.

Rodríguez, I. (2019). Intervención educativa para favorecer una convivencia pacífica e inclusiva: procesos y tendencias de cambio. Tesis de maestría. México: Universidad Pedagógica Nacional.

Rodríguez, L. (2009). La configuración de la formación de ciudadanía. Análisis de un caso en educación secundaria. México: Universidad Pedagógica Nacional.

Rojas, M. (2019). Deconstrucción de la risa en el contexto escolar. Entre la violencia y la convivencia de las y los adolescentes rumbo a la autonomía moral. Tesis de maestría. México: Universidad Pedagógica Nacional.

Romero, A. (2017). El uso del facebook para atender el tema de la discriminación con alumnos de segundo grado de secundaria. Tesis de maestría. México: Universidad Pedagógica Nacional.

Sánchez, D. (2016). El rincón del diálogo. Un camino hacia la educación para la paz y los derechos humanos. Tesis de maestría. México: Universidad Pedagógica Nacional.

Schön, D. (1998). El profesional reflexivo. Como piensan los profesores cuando actúan. Barcelona: Paidós.

Solís, N. (2019). Creación de espacios de diálogo y reconocimiento del otro para la transformación de conflictos. Hacia una Cultura de paz en el preescolar. Tesis de maestría. México: Universidad Pedagógica Nacional.

Sotelo, T. (2019). Aprendiendo a convivir, una experiencia de inclusión en el CAM. Tesis de maestría. México: Universidad Pedagógica Nacional. 
Tixtha, O. (2019). Formar para la autonomía, un desafío en la educación media superior. Tesis de maestría. México: Universidad Pedagógica Nacional.

Ugalde, R. (2017). Mediación entre pares. Una herramienta para la convivencia escolar. Tesis de maestría. México: Universidad Pedagógica Nacional.

Yurén, T. (2005). Ethos y autoformación en los dispositivos de formación de docentes. En Yurén, T. y Navia, C. (eds.) Ethos y autoformación en los dispositivos de formación de docentes. Barcelona: Pomares.

Yurén, T. y Mick, C. (2013). Educación y agencia. Aproximaciones teóricas y análisis de dispositivos. México: Juan Pablos Editor. 